Book Chapter

\title{
The Quantitative Criteria Based on the Fractal Dimensions, Entropy, and Lacunarity for the Spatial Distribution of Cancer Cell Nuclei Enable Identification of Low or High Aggressive Prostate Carcinomas
}

Przemyslaw Waliszewski ${ }^{1,2,3 *}$

${ }^{1}$ Department of Urology, Alb Fils Kliniken, Germany

${ }^{2}$ The Bȩdlewo Institute for Complexity Research, Poland

${ }^{3}$ Department of Urology, Ameos Clinics, Germany

*Corresponding Author: Przemyslaw Waliszewski, Department of Urology, Alb Fils Kliniken, Goeppingen, Germany

${ }^{\#}$ Current Address: Department of Urology, Ameos Clinics, Landrat-Beushausen Str 26, 31061 Alfeld, Germany

Published May 18, 2020

This Book Chapter is a republication of an article published by Przemyslaw Waliszewski at Frontiers in Physiology in February 2016. (Waliszewski P (2016) The Quantitative Criteria Based on the Fractal Dimensions, Entropy, and Lacunarity for the Spatial Distribution of Cancer Cell Nuclei Enable Identification of Low or High Aggressive Prostate Carcinomas. Front. Physiol. 7:34. doi: 10.3389/fphys.2016.00034)

How to cite this book chapter: Przemyslaw Waliszewski. The Quantitative Criteria Based on the Fractal Dimensions, Entropy, and Lacunarity for the Spatial Distribution of Cancer Cell Nuclei Enable Identification of Low or High Aggressive Prostate 
Carcinomas. In: Przemyslaw Waliszewski, Editor. Prime Archives in Physiology. Hyderabad, India: Vide Leaf. 2020.

(C) The Author(s) 2020. This article is distributed under the terms of the Creative Commons Attribution 4.0 International License(http://creativecommons.org/licenses/by/4.0/), which permits unrestricted use, distribution, and reproduction in any medium, provided the original work is properly cited.

Author Contributions: PW: all ideas, construction of the prostate data bank, digitalization of images, statistical analyses, writing of the manuscript.

Conflict of Interest Statement: The author declares that the research was conducted in the absence of any commercial or financial relationships that could be construed as a potential conflict of interest.

Acknowledgments: The author is grateful to Professor Michal Banaszak of Department of Physics, Adam-Mickiewicz University in Poznan, Poland and Professor John T. Isaacs, Department of Urology, The Johns Hopkins School of Medicine, Baltimore, USA for a discussion, minor suggestions, and editing.

This work was presented in part to the 15th Central European Meeting of the European Association of Urology in Budapest, Hungary on October 2-4, 2015.

\section{Abstract}

Background: Tumor grading, PSA concentration, and stage determine a risk of prostate cancer patients with accuracy of about $70 \%$. An approach based on the fractal geometrical model was proposed to eliminate subjectivity from the evaluation of tumor aggressiveness and to improve the prediction. This study was undertaken to validate classes of equivalence for the spatial distribution of cancer cell nuclei in a larger, independent set of prostate carcinomas. 
Methods: The global fractal capacity $D_{0}$, information $D_{1}$ and correlation $D_{2}$ dimension, the local fractal dimension (LFD) and the local connected fractal dimension (LCFD), Shannon entropy $H$ and lacunarity $\lambda$ were measured using computer algorithms in digitalized images of both the reference set $(n=60)$ and the test set $(n=208)$ of prostate carcinomas.

Results: Prostate carcinomas were re-stratified into seven classes of equivalence. The cut-off $D_{0}$-values $1.5450,1.5820$, $1.6270,1.6490,1.6980,1.7640$ defined the classes from $\mathrm{C} 1$ to C7, respectively. The other measures but the $D_{1}$ failed to define the same classes of equivalence. The pairs $\left(D_{0}, \mathrm{LFD}\right),\left(D_{0}, H\right)$, $\left(D_{0}, \lambda\right),\left(D_{1}, \mathrm{LFD}\right),\left(D_{1}, H\right),\left(D_{1}, \lambda\right)$ characterized the spatial distribution of cancer cell nuclei in each class. The coapplication of those measures enabled the subordination of prostate carcinomas to one out of three clusters associated with different tumor aggressiveness. For $D_{0}<1.5820$, LFD $<1.3$, LCFD $>1.5, H<0.7$, and $\lambda>0.8$, the class $\mathrm{C} 1$ or $\mathrm{C} 2$ contains low complexity low aggressive carcinomas exclusively. For $D_{0}>$ 1.6980, LFD $>1.7644$, LCFD $>1.7051, H>0.9$, and $\lambda<0.7$, the class $\mathrm{C} 6$ or $\mathrm{C} 7$ contains high complexity high aggressive carcinomas.

Conclusions: The cut-off $D_{0}$-values defining the classes of equivalence were validated in this study. The cluster analysis suggested that the number of the subjective Gleason grades and the number of the objective classes of equivalence could be decreased from seven to three without a loss of clinically relevant information. Two novel quantitative criteria based on the complexity and the diversity measures enabled the identification of low or high aggressive prostate carcinomas and should be verified in the future multicenter, randomized studies.

\section{Keywords}

Fractals, Complexity; Renyi Dimensions; Entropy; Lacunarity; Tumor Aggressiveness; Grading; Prostate Carcinoma 


\section{Synopsis}

The spatial distribution of cancer cell nuclei in digitalized histological images of prostate carcinomas reflects complex intercellular interactions within malignant tumor underlying its biological aggressiveness. Since this distribution can be characterized by a number of complexity and diversity measures, those measures define the quantitative criteria for low complexity carcinomas with low aggressiveness or for high complexity carcinomas with high aggressiveness.

\section{Introduction}

Histological evaluation of tumor aggressiveness, that is a potential of cancer cells for local growth and metastasis formation, is a key element in both diagnosis and prognosis of any malignancy. In the case of prostate carcinomas, pathologists developed about 40 subjective grading systems scaling aggressiveness of those tumors (reviewed in Humphrey, [1]). The Gleason system [2] is the most commonly used one [3]. It was validated with a database of a few thousand prostate cancer patients. Since its revision in 2005 and in 2010, the Gleason system comprises three patterns of tumor growth called the Gleason grades $[4,5]$. The Gleason score is calculated as a sum of two or, sometimes, three Gleason grades representing most predominant patterns of growth in a given carcinoma. This information co-determines the risk stratification of cancer patients. According to that stratification, cancer patients will undergo follow-up or will be treated. That latter option comprises a choice of the optimal therapy, extent of surgical resection with or without nerve sparing or dosing of radiation therapy [4-6].

A prostate biopsy is a procedure to obtain small samples of prostate tissue for the microscopic examination using a thin needle. Owing to both the limitations in obtaining a representative tissue specimen and a low specificity of this procedure, there is no good correlation between the grading score of the prostate carcinomas in biopsy tissues and in prostatectomy specimen [7]. Using a prostate biopsy, one cannot 
predict the existence of insignificant (indolent) cancer, that is cancer without a significant influence on survival [8], the final histological grade, size of tumor, its extracapsular extension, the existence of positive margins or lymph node involvement $[7,9,10]$. In addition, the modification of the criteria in the Gleason system $[4,5]$ caused both a discrepancy in tumor grading and difficulties in comparison of treatment results for patients treated before and after the change [6]. Although tumor aggressiveness is a key parameter in the prediction models, the subjectivity of histological evaluation is a source of the prediction weakness. Human eye is not able to evaluate correctly many details in tumor images, such as the ratio of cells bound in glands to the number of infiltrating cancer cells, or some configurations, such as the co-existence of glands of different size and shape. It is a challenge for pathologists to match the images to the definition of a grade, especially in the borderline cases (reviewed in Montironi et al., [11]). The subjective tumor grading has a significant inter- and intraobserver variability in the range of $38-80 \%$ [12-17] and the coefficient $\kappa$ for interobserver agreement $0.15-0.7$ [13,14,18-20]. Even though some DNA-, RNA-, or protein biomarkers were co-applied, it did not improve the accuracy of grading. The value of the correlation coefficient was low in the range of $0.1-0.6$ [21,22]. A novel diagnostic tool, prostate magnetic resonance imaging reinforced by the multiwavelet filters was supposed to enable the automated diagnosis of prostate carcinoma. The results remain nonspecific. Biopsies must be performed to evaluate tumor aggressiveness nonetheless (reviewed in Turkbey and Choyke, [23]).

A decision whether a prostate cancer patient should be treated or not depends on the fulfillment of two criteria [24-26]. The first one is the patient's overall life expectancy determined by age, health status, and comorbidities. In particular, patients older than 75 years, with life expectancy less than 10 years, in a reduced health status or with serious comorbidities will not be treated with the intention to cure. This category of patients will be offered a program of watchful waiting with a follow-up schedule. A palliative treatment can be initiated if local or metastatic progression with clinical symptoms occurs. The 
second criterion plays a pivotal role in the risk assessment of cancer progression. It considers three parameters [27], such as biological aggressiveness of tumor described by the subjective tumor grading according to Gleason [1-5], PSA concentration in serum [28-33], and tumor stage [34,35]. The statistical accuracy of the nomograms based on those parameters approximates $70 \%$ only $[27,31,36,37]$.

According to the clinical guidelines [24-26], low risk cancer patients with PSA $<10 \mathrm{ng} / \mathrm{ml}$, Gleason score of 6 or less, and pT1-2a stage do not need any treatment, nor an adjuvant therapy. Despite of those criteria, the overtreatment occurs at the indolent stadium of the disease in about 55\% of patients [37-39]. Urologists recommend frequently treatment even for 80 -year-old patients with limited life expectancy [38-41]. Rather, those patients should be followed-up to identify local tumor progression and aggressive metastatic disease in the long term [40-43]; phenomena especially frequent about 15 years from the diagnosis [44-47]. The same strategy might also be offered to some patients with intermediate risk of progression (PSA 10-20 $\mathrm{ng} / \mathrm{ml}$, Gleason score 7, pT2b-2c) [45]. Unfortunately, only up to $49 \%$ of eligible patients participate in the follow-up programs [48]. The lack of the histological, molecular or clinical criteria for the unequivocal identification of the low risk cancer patients increases a fear for tumor progression among those involved in active surveillance as well as among those who already underwent some kind of aggressive therapy, without even knowing if they needed it [48].

Patients with high-risk (PSA $>20 \mathrm{ng} / \mathrm{ml}$, Gleason score 8-10, stage T3a) or very high risk carcinomas (stage T3b-T4 N0 or any $\mathrm{T}$ N1) should be treated aggressively with all available modalities, such as surgery, radiotherapy, brachytherapy, or hormonal therapy, even though a choice of a modality seems to increase survival in about $10 \%$ of patients [49]. For example, SEER, a population-based cohort study of 66,717 patients 66 years of age or older with localized prostate cancer demonstrated that primary hormonal therapy did not improve long-term overall or disease-specific survival [50]. 
The relevant question, whether a given prostate carcinoma is an indolent, low grade, low risk malignancy rather than a high grade, high risk one can be answered only if prostate gland is removed surgically. Although this answer appears after the initiation of the aggressive treatment, it is still important to get the unequivocal evaluation of tumor aggressiveness based on some objective, quantitative criteria. Those criteria might help to improve the accuracy of prognosis, to plan more individualized therapy, or to facilitate a search for some features of cancer cells that would improve predictions made on the basis of biopsies. A variety of approaches, such as graph models [51], gland segmentation with morphometric analysis and application of probabilistic pairwise Markov models [52-54], color channel histograms, texture analysis, fractal algorithm with fractal code or multifeature analysis with a number of classifiers, such as Bayesian one [55], multiwavelet method [56,61], k-NN one [62], support vector machine [63], or linear discriminant analysis method $[62,63]$ were proposed for the automated diagnosis of prostate carcinoma or for the evaluation of tumor aggressiveness. Those statistically elegant and mathematically advanced approaches resulted in the maximal accuracy of $78-81 \%$ for grading in the case of multiwavelet method $[62,63]$. The accuracy of 95-97\% was reported only for the low number of carcinomas [61]. This discrepancy between the Gleason grading and the quantitative classifications generated by the sophisticated computer algorithms had at least one reason. Two different categories of data, the subjective and the objective one, should not be compared each other if the subjective tumor grading according to Gleason is chosen as the absolute frame of reference.

Since there is no golden standard in pathology of adenocarcinomas that might be used as the objective frame of reference for the quantitative studies, a novel approach based on the fractal geometric model of prostate carcinomas and evaluation of complexity in the spatial distribution of cancer cell nuclei was proposed [64-66]. Cell nuclei are fragments of the surface in two-dimensional histological images. They compose irregular, yet self-affine configurations [64]. The fractal geometrical model of prostate carcinomas introduced the idea of 
the distinct classes of equivalence called complexity classes [66]. Those classes were defined by the values of the global capacity fractal dimension $D_{0}$. Using that model, all basic patterns of growth seen in prostate cancer were subordinated to the appropriate class. In particular, low grade prostate carcinomas with well-preserved glandular structure were re-stratified to the class $\mathrm{C} 1, \mathrm{C} 2$, or $\mathrm{C} 3$. High grade carcinomas with the predominating infiltrating patterns of growth were stratified to the class $\mathrm{C} 6$ or $\mathrm{C} 7$.

This study was undertaken to validate the classes of equivalence [66] using utterly novel set of carcinomas than that used in the study [64-66]. A question arises whether the additional complexity or diversity measures, such as the local fractal dimensions, Shannon entropy or lacunarity allow a similar stratification of prostate carcinomas into the same classes of equivalence? Is it possible to define some quantitative criteria based on all those parameters that define prostate carcinomas with low or high complexity of the spatial distribution of cancer cell nuclei associated with low or high tumor aggressiveness?

\section{Methods}

\section{The Renyi Family of the Global Fractal Dimensions}

The Renyi family of the global spatial fractal dimensions comprises the capacity $\left(D_{0}\right)$, information $\left(D_{1}\right)$, and correlation $\left(D_{2}\right)$ dimension. The appropriate introduction to the theory of dimension, fractals, or the formal mathematical definitions of the fractal dimensions can be found elsewhere $[67,68]$. Briefly, the capacity dimension $D_{0}$ is defined as a relationship between the logarithm of a number of boxes $N(\varepsilon)$ covering the geometric object and the logarithm of a box size $\varepsilon$ (Equation 1; [69,70]):

$$
D_{0}=\lim _{\varepsilon \rightarrow 0} \frac{\log N(\varepsilon)}{\log \frac{1}{\varepsilon}}
$$

The information dimension $D_{1}$ measures how the average information needed to identify an occupied box, scales, as the 
scale of boxes gets smaller. The algorithm for the information dimension will search for the linear relationship between the logarithm of a box size $(\varepsilon)$ and the logarithm of the probability $p$ that a given box contains the element of the object (Equation 2; Baker and Gollub, [71]).

$$
D_{1}=\lim _{\varepsilon \rightarrow 0} \frac{-\left\langle\log p_{\varepsilon}\right\rangle}{\log \frac{1}{\varepsilon}}
$$

The correlation dimension $D_{2}$ measures the number of points $M$ used to generate a representation of the fractal and the number of pairs of points closer than $\varepsilon$ to each other. The correlation dimension is a probability measure that two pixels within the object are close to each other less than $\varepsilon$. The appropriate algorithm calculates not only if a given box is occupied by the pixel of the analyzed geometric object, but also how many pixels in the box are, and how close to each other they are (Equation 3):

$$
D_{2}=\lim _{\varepsilon \rightarrow 0, M \rightarrow \infty} \frac{\log \left(g_{\varepsilon} / M^{2}\right)}{\log \varepsilon}
$$

in which $M$ is a number of pixels of the analyzed geometric object and $g_{\varepsilon}$ denotes a number of pairs of pixels that are closer to each other than $\varepsilon[71,72]$.

\section{Local Connected Fractal Dimension}

The local fractal dimension (LFD) is the fractal capacity dimension calculated according to Equation 1 for every pixel in the image. This dimension is a local and indirect measure of intercellular connectivity, i.e., interconnectedness which denotes the existence of complex, dynamic relationships in a population of cells leading to the spatial and temporal emergence of global features in the system that would never appear in a single cell existing out of the system [73-78]. 
The local connected fractal dimension (LCFD) characterizes local irregularities of geometry of heterogeneous geometrical objects, such as clusters of cancer cells present either in glands or in infiltrates. Instead of a single value of the global fractal capacity dimension $D_{0}$ calculated for the entire image, one gets a set of values calculated for each pixel that belongs to the analyzed object. This is done according to the following Image $\mathbf{J}$ algorithm:

Step 1: choose a pixel $\mathrm{P}$ that belongs to the analyzed object and possesses eight neighbor pixels.

Step 2: define the local connected set of pixels by finding all the pixels connected to the pixel $\mathrm{P}$ within the increasing s-pixel-side window centered at $P$.

Step 3: count how many pixels $\mathrm{N}(\mathrm{s})$ of the analyzed object are within the window.

Step 4: use the least square method to compute the slope of the $\log -\log$ curve composed by the coordinates $[\log (\mathrm{N}(\mathrm{s}), \log (\mathrm{s})$; Landini et al., [79]].

\section{Entropy and the Global Information Fractal Dimension $D_{1}$}

The Shannon entropy $H$ is a statistical measure of both the information content and diversity in a given cell configuration in the image. It can be used to characterize gray images or binary images of the spatial distribution of cancer cell nuclei. The Shannon entropy describes the following Equation (4):

$$
H=-\sum_{i} p_{i} \log _{2} p_{i}
$$

In the above equation, $p_{i}$ denotes the probability that the difference between two adjacent pixels of the image is equal to $i$, and is calculated from the histogram counts. Low entropy images, such as those containing mostly black background, e.g., in benign prostatic hyperplasia or in well-differentiated adenocarcinomas, have large numbers of pixels with the same 
zero value. The black image that contains no white pixels will have entropy equal zero.

The entropy $H_{D}$ of a set with $D$ dimensions is defined by the following Equation (5):

$$
H_{D}=-\sum_{i} p_{i} \log _{2} p_{i}+D \log \varepsilon=H+D \log \varepsilon
$$

ín which $H$ stands for the Shannon entropy (see Equation 4), $\varepsilon$ denotes size. In consequence, entropy $H$ is a linear function of $\log \varepsilon$ with slope $D$ and intersection $H_{D}$. It is important to notice that the slope $D$ in the limit for $\varepsilon=0$ becomes the global information fractal dimension $D_{1}$ (see Equation 2; Sethna, [80]).

\section{Lacunarity}

Cancer cells form various morphological patterns. The areas of the image complementary to the lumen of glands correspond to the gaps on the binary image. Investigating those gaps provides important information about homogeneity of cell distribution in malignant tumor. Lacunarity $\lambda$, that is, a degree of gappiness, inhomogeneity, or translational and rotational invariance in the image characterizes quantitatively those gaps as well as differences in their spatial distribution between the patterns of growth. This parameter can also quantify differences between self-similar structures having the same global fractal dimension [81].

In general, lacunarity $\lambda$ is equivalent to the square of the coefficient of variation, $\mathrm{CV}$, and expresses a relationship between standard deviation $\sigma$ and mean $\mu$ for the pixels in a given area of an image, and a number of pairs of pixels $g$ that are closer to each other than $\varepsilon$ :

$$
\lambda_{\epsilon, g}=\left(C V_{\epsilon, g}\right)^{2}=\left(\frac{\sigma_{\epsilon, g}}{\mu_{\epsilon, g}}\right)^{2}
$$

More specifically, lacunarity $\lambda$ is a relationship between variation for the function $P(m, r)$, that defines probability of the 
localization of the pixels $m$ in the square of size $r$ to the square of the mean value of that function (Equation 7):

$$
\lambda(r)=\frac{\sum_{m=1}^{r^{2}} m^{2} P(m, r)-\left(\sum_{m=1}^{r^{2}} m P(m, r)\right)^{2}}{\left(\sum_{m=1}^{r^{2}} m P(m, r)\right)^{2}}
$$

The computer algorithms that calculate the mean value of lacunarity for images use the same principle as in the case of the global fractal dimensions, i.e., they analyze the digital image from different scaled levels of resolution to examine how certain geometric features change with the size of the element used to inspect the image. The first approach, called box counting, is identical with the principle underlying the capacity dimension (see above) in the sense that the box for each $\varepsilon$ is placed as though it were part of a grid overlaid on the image so that the box does not overlap itself. In the second one, called the sliding box algorithm, the box is slid over the image so that it overlaps itself and the sliding box lacunarity is calculated. The values are usually expressed as logarithms. Lacunarity analyses using the types of values discussed above have shown that data sets extracted from geometric fractals, or from patterns that change little when rotated have low lacunarity. The larger and more irregular are the gaps in the image, as those seen in glands in benign prostatic hyperplasia, the greater is the value of lacunarity. In some instances, fractal dimensions and values of lacunarity can be correlated, but it does not hold for all types of patterns and measures of lacunarity.

\section{Patients, Preparation of Images, and Isolation of Cell Nuclei}

This study was performed according to the ethical standards outlined in the WMA Declaration of Helsinki "Ethical Principles for Medical Research Involving Human Subjects" (http://www.wma.net). The data bank was constructed and analyzed according to the ethical requirements of the Ethics Committee for the Medical Faculty of the Justus-Liebig University in Giessen, Germany (ethical vote 49/05). Since this project was based solely on computer-aided image analysis of 
the digitalized anonymous tissue slides of prostate carcinomas, no written consent from the prostate cancer patients was necessary. The surgical procedures were performed from 2007 to 2010. Tissues were stained with hematoxylin and eosin as well as with the appropriate antibodies against the markers, i.e., PSA (DAKO, Germany) and AMACR (DAKO, Germany) for prostate cancer cells, cytokeratin 34ßE12 (Leica Biosystems, Canada) for basal cells in order to confirm the diagnosis [82], and classified by the Gleason criteria [1-5].

A reference set of prostate carcinomas comprised cases representing the homogeneous patterns with the Gleason score $3+3=6$ defined as a regular gland architecture with no basal cells and no areas of cellular infiltration $(n=20)$, the Gleason score $4+4=8$ defined as dominating small glands spread freely in the prostate tissue or organized in the cords $(n=20)$, and the Gleason score $5+5=10$ defined as a random infiltration of the prostate tissue by cancer cells without any glands $(n=20)$, and benign prostatic hyperplasia $(n=10)$. We analyzed and restratified a test set of prostate carcinomas comprising 208 carcinomas with the following Gleason score: $3+3=6(n=70)$, $3+4=7 a(n=18), 4+3=7 b(n=14), 4+4=8(n=23), 4+5=9$ $(n=28), 5+4=9(n=20), 5+5(n=35)$. All prostate carcinomas were subordinated to the appropriate subsets called structural Gleason classes using the Gleason criteria. We also analyzed benign prostatic hyperplasia $(n=20)$. None of the patients had lymphatic or distant metastases.

Histological slides of prostate carcinomas stained with hematoxylin and eosin were digitalized using both a microscope Axioskop 5.0 with the halogen lamp 12V 50W 2800K $950 \mathrm{~lm}$ and the objective Plan Neofluar 20x with the numerical aperture 0.5 , Zeiss, Germany and a camera Nikon Coolscope, Japan. The optimal conditions for the magnification (20x) and lighting intensity $\left(41.19 \times 10^{6} \mathrm{~lx}\right)$ were chosen in such a manner that the values of the capacity fractal dimension for the test image were in the plateau-area of the test curve (data not shown).

The color images in the bmp format with $1240 \times 1000$ pixels and resolution $150 \times 150$ dpi were first resized to the jpg format with 
$648 \times 432$ pixels and resolution $150 \times 150$ dpi. Cancer cell nuclei were isolated electronically using a package Definiens Tissue Map ver. 7.0 (Definiens, Munich, Germany), and stored as RPG images in the jpg format with $648 \times 432$ pixels, resolution $150 \times 150 \mathrm{dpi}$. Both conversion to the eight-bit images and their thresholding to the binary images, i.e., images having only 0 (black) and 255 (white) pixel values was performed using the Renyi Entropy filter of the open-source software Image $\mathbf{J}$ ver $1.48 \mathrm{v}$ (NIH, Bethesda, USA, http://imagej.nih.gov/ij/). The images were analyzed by the computer algorithms measuring the global fractal dimensions of the Renyi family, i.e. capacity dimension $D_{0}$ and information dimension $D_{1}$ (Benoit 1.3, True Soft, USA, http://www.trusoft-international.com/benoit.html), and correlation dimension $D_{2}$ (Fractalyse 2.4, CNRS Universite de Franche-Comte, France, http://www.fractalyse.org). The values of the global fractal dimensions generated by the computer algorithms were verified against the geometrical model [64-66]. Both the local fractal dimension and the local connected fractal dimension were measured in the same digitalized images using the open-source software Image $\mathrm{J}$ ver $1.48 \mathrm{v}$ and opensource plugin FracLac 2013 Janb420 by A. Karperien (http://imagej.nih.gov/ij/plugins).

\section{Statistical Analysis}

The statistical analysis including the ROC analysis was performed by Sigma Plot ver. 10.0 (Systat Software Inc., San Jose, USA, http://www.sigmaplot.com). The cluster analysis was performed by Mathematica ver. 10.0 (Wolfram Research Inc., USA) and Statistica ver. 8.0 (Statsoft, Oklahoma City, USA). The following aspects are important while analyzing a ROC curve. The ROC curve is a two dimensional graph. It represents a relationship between sensitivity of a statistical test (y-axis) in a function of 1-specificity of that test ( $\mathrm{x}$-axis). Sensitivity is defined as a rate of true positive events. The second quantity represents a rate of non-events that were falsely identified as positive events for the different cut-off values of a binary classifier, such as $D_{0}, D_{1}$, or $D_{2}$, LFD, etc. Second, the closer the ROC curve follows the y-axis, and then the top border of the ROC diagram, the more accurate the statistical test. The area 
under the ROC curve (AUC) is a probability of the test accuracy, i.e., a probability that the classifier discriminates between the elements of two sets correctly. In particular, the AUC of 1.0 denotes a perfect relationship between the data that belong to a given category and those that do not. Third, the closer the ROC curve comes to the diagonal of the ROC diagram, the AUC is closer 0.5 , what denotes that the test is not able to discriminate between two sets of data. It should be noted that a task of the restratification of prostate carcinomas on the basis of the quantitative characteristics of the spatial distribution of cancer cell nuclei by the fractal dimensions $D_{0}, D_{1}$, and $D_{2}$ into the complexity classes is equivalent to the task of the identification the cut-off values of the best classifier, i.e., the classifier generating the AUC of 1.0 [83].

Cluster analysis is a data mining tool that uses a number of computer algorithms and statistical methods to sort elements of a set into groups (clusters) in such a manner that the elements belonging to the same cluster have a maximal degree of association and a minimal degree otherwise. In this work, a $v$ fold cross-validation algorithm was applied to determine the number of clusters in the data. Then, the $k$-means algorithm was applied to produce exactly $k$ different clusters predicted by the first algorithm with the greatest possible distinction and maximal distances between those clusters. One examines here the means for each cluster on each dimension (parameter) to assess how distinct the $k$-means clusters are. Ideally, the means for all dimensions should be different, and the magnitude of the $F$ values from the analysis of variance performed on each dimension indicates how well the dimensions discriminate between clusters [83].

\section{Results}

\section{The Global Capacity Fractal Dimension $\left(D_{0}\right)$ and the Information Fractal Dimension $\left(D_{1}\right)$ Discriminate Well between the Structural Gleason Classes (Grades)}

Both the global capacity and information fractal dimension, $D_{0}$ and $D_{1}$, have the sufficient statistical power to discriminate between the Gleason classes, that is the subsets of prostate 
carcinomas with the Gleason score $3+3,4+4$, or $5+5$. Figures 1 , 2 demonstrate that the dimension $D_{0}$ was slightly better than the dimension $D_{1}$ (Figure 1) and much better than the global correlation fractal dimension $D_{2}$. In that latter case, the area under the curve (AUC) was almost 1.00 for the dimension $D_{0}$ vs. 0.8 for the dimension $D_{2}$. In addition, coefficient of correlation between the $D_{0}$ and $D_{1}$ was very high $R=0.963$. The local fractal dimension, LFD, its variance, the local connected fractal dimension, LCFD, its variance, entropy $H$, and lacunarity $\lambda$ were not able to discriminate between those classes at the statistically significant level (data not shown).

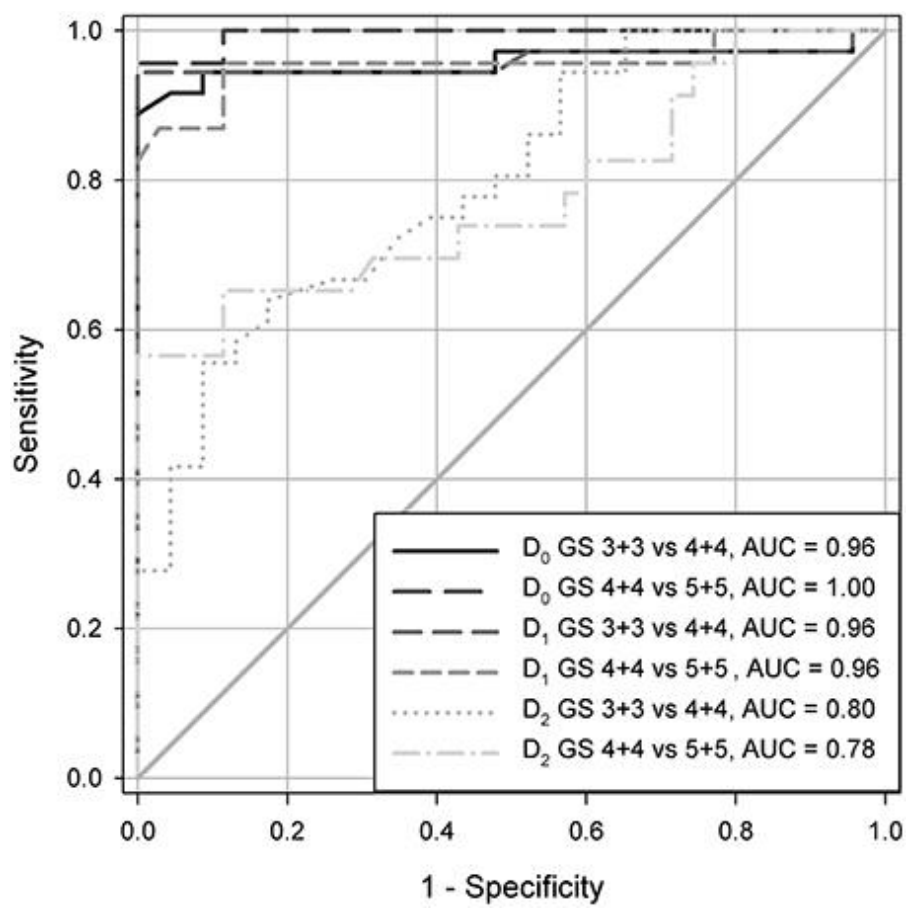

Figure 1: The ROC analysis of prostate carcinomas with Gleason score 3+3, $4+4$, and $5+5$ of the test set demonstrating a discriminating power of the fractal dimensions $D_{0}, D_{1}$, and $D_{2}$. AUC stands for the area under the curve, and has values in a similar range as in the case of the reference set. 
Prime Archives in Physiology

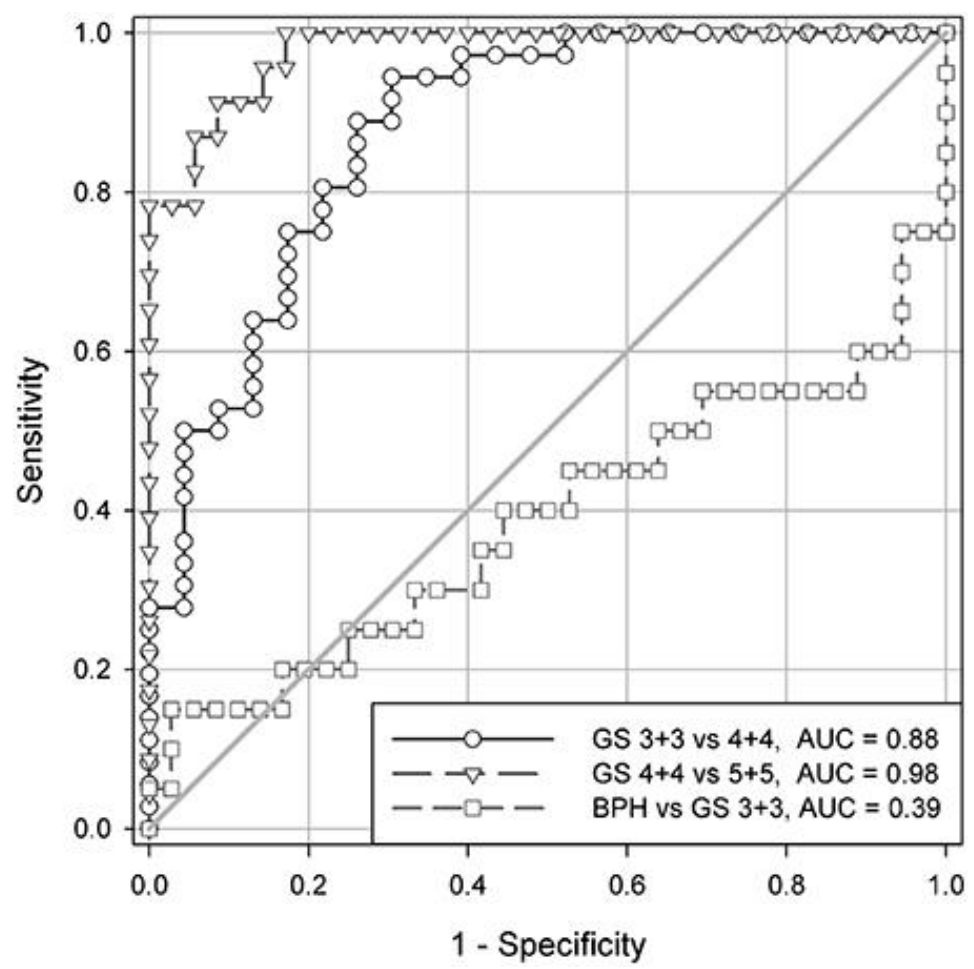

Figure 2: The ROC analysis of carcinomas with Gleason score $3+3,4+4$, and $5+5$ of the test set demonstrating a discriminating power of the local fractal dimensions LFD. AUC stands for the area under the curve, and has values in a similar range as in the case of the reference set.

It is worth to notice that none of the complexity or the diversity measures can discriminate between the spatial distribution of cell nuclei in benign prostatic hyperplasia and in prostate carcinomas of the complexity class $\mathrm{C} 1$ containing carcinomas with the Gleason score 3+3 (compare Tables 1, 3, and Figure 2). The mean values overlap (Table 3 ), and differences between those two groups of tissues are not statistically significant ( $t$-Student test, $p>0.1$. 
Prime Archives in Physiology

Table 1: Results of ROC analysis for the reference set (A) or the test set (B) of prostate carcinomas and their stratification into the structural Gleason classes.

\begin{tabular}{|c|c|c|c|c|c|}
\hline Gleason classes & Sensitivity & Specificity & Cut off-value $D_{0}$ & AUC & $p$-value \\
\hline \multicolumn{6}{|l|}{ (A) REFERENCE SET } \\
\hline BPH vs. Gleason $3+3$ & 0.50 & 0.40 & 1.5050 & 0.48 & 1.10 \\
\hline Gleason $3+3$ vs. $4+4$ & 1.00 & 1.00 & 1.6040 & 1.00 & $<0.0001$ \\
\hline Gleason $4+4$ vs. $5+5$ & 1.00 & 1.00 & 1.6910 & 1.00 & $<0.0001$ \\
\hline \multicolumn{6}{|l|}{ (B) TEST SET } \\
\hline BPH vs. Gleason $3+3$ & 0.50 & 0.51 & 1.5130 & 0.50 & 0.96 \\
\hline Gleason $3+3$ vs. $3+4$ & 0.63 & 0.67 & 1.5290 & 0.64 & $<0.09$ \\
\hline Gleason $3+4$ vs. $4+3$ & 0.78 & 0.67 & 1.5580 & 0.79 & 0.008 \\
\hline Gleason $4+3$ vs. $4+4$ & 0.75 & 0.78 & 1.6160 & 0.85 & 0.0008 \\
\hline Gleason $4+4$ vs. $4+5$ & 0.56 & 0.50 & 1.6400 & 0.47 & 1.27 \\
\hline Gleason $4+5$ vs. $5+4$ & 0.86 & 0.70 & 1.6870 & 0.84 & $<0.0001$ \\
\hline Gleason $5+4$ vs. $5+5$ & 0.80 & 0.66 & 1.7610 & 0.83 & $<0.0001$ \\
\hline
\end{tabular}

\section{The Gleason Classes and Complexity Classes are not Identical}

Table 1 contains results of ROC analysis for two sets of prostate carcinomas. The first one is a reference set. It contains carefully selected carcinomas with relatively homogeneous structure. Those carcinomas were stratified into three distinct structural classes with the Gleason score $3+3,4+4$, or $5+5$. The spatial distribution of cancer cell nuclei in those carcinomas was also characterized quantitatively by the global fractal dimensions $D_{0}$, $D_{1}$, and $D_{2}$. The ROC analysis enabled calculation of the cut-off $D_{0}$-values that defined the numerical limits of those structural Gleason classes (see Table 1). As it turned out, even very careful histological evaluation of tumor structure brought together cases with a variety of infinitesimal structural alterations that could not be defined unambiguously. In consequence, each structural Gleason class of the reference set contained a low number of carcinomas with the quantitative characteristic typical of the other classes. Those cases were re-stratified using the cut-off $D_{0^{-}}$ values for the structural Gleason classes as a frame of reference. After that operation, the new cut-off $D_{0}$-values were calculated. They define the complexity classes $\mathrm{C} 1, \mathrm{C} 4$, and $\mathrm{C} 7$ (compare Table 1A with Table 2A). 
Table 2: Results of the ROC analysis for the re-stratified reference set (A) or the re-stratified test set (B) of prostate carcinomas.

\begin{tabular}{|c|c|c|c|c|c|}
\hline Complexity classes & Sensitivity & Specificity & Cut off-value $D_{0}$ & AUC & $p$-value \\
\hline \multicolumn{6}{|l|}{ (A) REFERENCE SET } \\
\hline C1 vs. C4 & 1.00 & 1.00 & 1.5980 & 1.00 & $<0.0001$ \\
\hline C4 vs. C7 & 1.00 & 1.00 & 1.7000 & 1.00 & $<0.0001$ \\
\hline \multicolumn{6}{|l|}{ (B) TEST SET } \\
\hline C1 vs. C2 & 1.00 & 1.00 & 1.5450 & 1.00 & $<0.0001$ \\
\hline $\mathrm{C} 2$ vs. C3 & 1.00 & 1.00 & 1.5820 & 1.00 & $<0.0001$ \\
\hline C3 vs. C4 & 1.00 & 1.00 & 1.6270 & 1.00 & $<0.0001$ \\
\hline C4 vs. C5 & 1.00 & 1.00 & 1.6490 & 1.00 & $<0.0001$ \\
\hline C5 vs. C6 & 1.00 & 1.00 & 1.6980 & 1.00 & $<0.0001$ \\
\hline C6 vs. C7 & 1.00 & 1.00 & 1.7640 & 1.00 & $<0.0001$ \\
\hline
\end{tabular}

The Cut-off $D_{0}$-values of the Structural Gleason Classes $3+4,4+3,4+5$, or $5+4$ are Located between the Values for the Structural Gleason Classes $3+3,4+4$, or $5+5$

The test set contains prostate carcinomas representing all Gleason scores. Those carcinomas were chosen randomly from the archive (Table 1B). The cut-off $D_{0}$-values for the structural classes Gleason $3+3,4+4$, or $5+5$ in that set are close to those of the reference set if analyzed without the structural classes $3+4$, $4+3,4+5$, and 5+4. The same is true of the AUC values (Figures $1,2)$ as well as sensitivity and specificity (data not shown). It appears that human eye deals much better with the evaluation of the distinct homogeneous structures rather than with the heterogeneous ones, where the number of details is greater, more difficult to grasp, and to set in order.

If the structural classes $3+4,4+3,4+5$, and $5+4$ are taken into consideration, the cut-off $D_{0}$-values locate them between the values for the structural classes Gleason $3+3,4+4$, or $5+5$. The $D_{0}$ intervals defining those heterogeneous classes are smaller now (compare with Tables 1A,B). However, both sensitivity and specificity are not equal 1.00. The AUC values are also far from 1.0. This is because all structural classes of the test set including those with seemingly homogeneous carcinomas contain some number of cases with complexity measures typical of the other structural Gleason classes. As expected, those "impure" cases were re-stratified according to the $D_{0}$-cut-off values, as described in the next paragraph, to the adjacent complexity classes exclusively. 


\section{Re-Stratification of Prostate Carcinomas into the Seven Classes of Equivalence}

Re-stratification puts aside a problem of accuracy of the subjective evaluation as well as troubles connected with a matching of tumor structure under examination to the grade definition. This was achieved by the application of the cut-off $D_{0}$-values of the re-stratified reference set (see Table 2A). Carcinomas with the quantitative characteristics that did not match well the characteristics of the other carcinomas of the same class, nor of the adjacent class were identified and clustered in the novel classes of equivalence defined by the novel $D_{0}$-values. As a result, carcinomas of the test set were restratified to the seven classes of complexity, i.e., C1, C2, C3, C4, C5, C6, and C7 (Table 2B). Table 3 provides a percentage of cases of each structural Gleason class that was a subject to restratification. This was from 30 to $70 \%$ of cases per structural class.

It should be emphasized that all complexity classes are unequivocally defined by the cut-off $D_{0}$-values. Therefore, the complexity classes contain only those prostate carcinomas that have the spatial distribution of cancer cell nuclei characterized by the similar values of the dimension $D_{0}$ and hold a condition that the AUC in the ROC analysis equals 1.0. As expected, an identical re-stratification can be obtained with $D_{1}$-values (compare Figure 3B). Each complexity class contains elements of the corresponding structural Gleason class and some carcinomas of the other structural Gleason classes with the $D_{0^{-}}$ values within the range for that class. For example, Figure $3 \mathrm{~A}$ presents each carcinoma of the test set as a point on the $2 \mathrm{D}$ scatter plot with the $D_{0}$ and the LFD as variables. Each carcinoma belongs to one out of seven classes of equivalence according to the $D_{0}$-values. Table 3 shows the statistically significant differences between all complexity classes for the $D_{0}$, and the $D_{1}(p<0.0001, t$-Student test). This finding confirms a choice of the cut-off $D_{0}$ or $D_{1}$-values as a criterion for the restratification. 
Prime Archives in Physiology

Table 3: The values of the mean, median and standard deviations for the complexity or diversity measures in the re-stratified test set of prostate carcinomas.

\begin{tabular}{|c|c|c|c|c|c|c|c|c|c|}
\hline & & \multirow{2}{*}{$\begin{array}{l}\text { Staging Grading } \\
\text { PSA ng/ml } \\
\text { Gleason Class }\end{array}$} & \multirow{2}{*}{$\begin{array}{l}\text { pT1b - pT2a } \\
\text { Gleason } 6 \\
\text { PSA < } 10 \\
\text { GS } 3+3\end{array}$} & \multicolumn{3}{|c|}{ pT2b-pT2cGleason 7-810 < PSA $<20$} & \multicolumn{3}{|c|}{ pT3a-pT3bGleason 9-10PSA > 20} \\
\hline & & & & GS $3+4$ & GS $4+3$ & GS $4+4$ & GS $4+5$ & GS $5+4$ & GS $5+5$ \\
\hline & & & $n=70$ & $n=18$ & $n=14$ & $n=23$ & $n=28$ & $n=20$ & $n=35$ \\
\hline \multicolumn{2}{|c|}{$\begin{array}{l}\text { Complexity/Diversity } \\
\text { Measures }\end{array}$} & BPH & Class C1 & Class C2 & Class $\mathrm{C} 3$ & Class $\mathrm{C} 4$ & Class C5 & Class $\mathrm{C} 6$ & Class $\mathrm{C7}$ \\
\hline & & $n=20$ & $n=60$ & $n=20$ & $n=37$ & $n=16$ & $n=23$ & $n=29$ & $n=23$ \\
\hline \multirow[t]{3}{*}{$D_{0}$} & Mean & 1.5038 & 1.4836 & 1.5659 & 1.6035 & 1.6383 & 1.6684 & 1.7318 & 1.7986 \\
\hline & Median & 1.5154 & 1.4895 & 1.5665 & 1.6023 & 1.6378 & 1.6660 & 1.7287 & 1.7944 \\
\hline & SD & 0.0902 & 0.0490 & 0.0125 & 0.0125 & 0.0066 & 0.0138 & 0.0199 & 0.0241 \\
\hline \multirow[t]{3}{*}{$D_{1}$} & Mean & 1.5222 & 1.5114 & 1.5673 & 1.6081 & 1.6474 & 1.6703 & 1.7282 & 1.7651 \\
\hline & Median & 1.5321 & 1.5190 & 1.5700 & 1.6029 & 1.6403 & 1.6620 & 1.7286 & 1.7570 \\
\hline & SD & 0.0936 & 0.0445 & 0.0250 & 0.0247 & 0.0232 & 0.0260 & 0.0221 & 0.0237 \\
\hline \multirow[t]{3}{*}{$D_{2}$} & Mean & 1.5342 & 1.5525 & 1.6426 & 1.6657 & 1.7046 & 1.7069 & 1.7575 & 1.8107 \\
\hline & Median & 1.5488 & 1.5640 & 1.6466 & 1.6687 & 1.6964 & 1.7144 & 1.7625 & 1.7993 \\
\hline & SD & 0.0734 & 0.0977 & 0.0634 & 0.0586 & 0.0641 & 0.0511 & 0.0357 & 0.0452 \\
\hline \multirow[t]{3}{*}{ LFD } & Mean & 1.6310 & 1.5109 & 1.5396 & 1.6529 & 1.6992 & 1.6897 & 1.7644 & 1.8225 \\
\hline & Median & 1.6310 & 1.5564 & 1.5751 & 1.6492 & 1.6946 & 1.6998 & 1.7716 & 1.7989 \\
\hline & SD & 0.0792 & 0.1447 & 0.2129 & 0.1497 & 0.1342 & 0.1236 & 0.0821 & 0.0552 \\
\hline CV & Mean & 0.0939 & 0.0631 & 0.0582 & 0.0594 & 0.0761 & 0.0817 & 0.0780 & 0.0450 \\
\hline \multirow[t]{2}{*}{ LFD } & Median & 0.0829 & 0.0677 & 0.0370 & 0.0466 & 0.0580 & 0.0629 & 0.0696 & 0.0450 \\
\hline & SD & 0.0357 & 0.0253 & 0.0507 & 0.0453 & 0.0622 & 0.0502 & 0.0356 & 0.0144 \\
\hline \multirow[t]{3}{*}{ LCFD } & Mean & 1.4507 & 1.4908 & 1.5716 & 1.6106 & 1.5663 & 1.6310 & 1.7051 & 1.7212 \\
\hline & Median & 1.5223 & 1.5256 & 1.5625 & 1.6351 & 1.5815 & 1.6401 & 1.7369 & 1.7348 \\
\hline & SD & 0.2063 & 0.1353 & 0.1665 & 0.2110 & 0.2639 & 0.1935 & 0.1313 & 0.1432 \\
\hline CV & Mean & 0.1916 & 0.1403 & 0.1421 & 0.1074 & 0.1462 & 0.1002 & 0.1085 & 0.1154 \\
\hline \multirow[t]{2}{*}{ LCFD } & Median & 0.1724 & 0.1261 & 0.1037 & 0.0973 & 0.1664 & 0.0944 & 0.0898 & 0.1178 \\
\hline & SD & 0.0514 & 0.0473 & 0.0526 & 0.0671 & 0.0831 & 0.0687 & 0.0543 & 0.0609 \\
\hline \multirow[t]{3}{*}{$H$} & Mean & 0.5760 & 0.5638 & 0.6946 & 0.7594 & 0.7866 & 0.8457 & 0.9361 & 0.9500 \\
\hline & Median & 0.5775 & 0.5803 & 0.7007 & 0.7504 & 0.7944 & 0.8451 & 0.9369 & 0.9861 \\
\hline & SD & 0.1234 & 0.1072 & 0.0376 & 0.0337 & 0.0533 & 0.0219 & 0.0253 & 0.0661 \\
\hline \multirow[t]{3}{*}{$\lambda$} & Mean & 0.8516 & 0.8597 & 0.8125 & 0.7656 & 0.7527 & 0.7205 & 0.6284 & 0.6108 \\
\hline & Median & 0.8515 & 0.8552 & 0.8141 & 0.7661 & 0.7507 & 0.7210 & 0.6095 & 0.5678 \\
\hline & SD & 0.0524 & 0.0370 & 0.0215 & 0.0309 & 0.0367 & 0.0183 & 0.0405 & 0.0843 \\
\hline
\end{tabular}



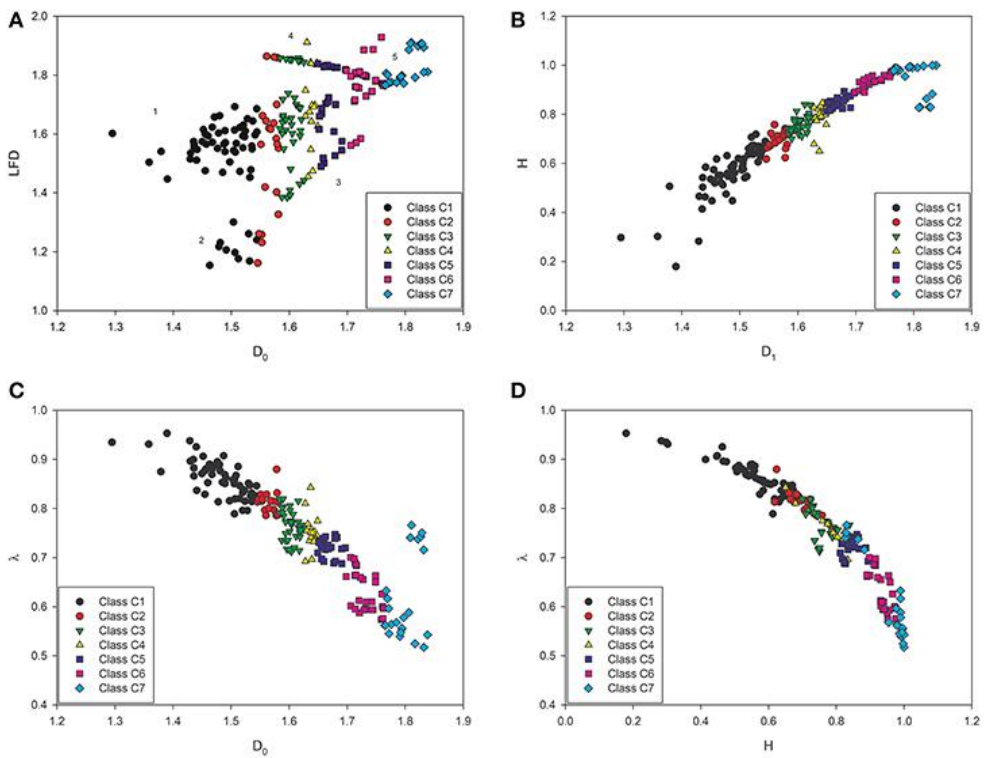

Figure 3: Two dimensional scatter plots showing all prostate carcinomas of the test set stratified according to a number of relationships between the parameters: (A) $\left(D_{0}, \mathrm{LFD}\right),(\mathrm{B})\left(D_{1}, H\right),(\mathrm{C})\left(D_{0}, \lambda\right)$, and (D) $(H, \lambda)$. Differences between the complexity classes defined by the $D_{0}$ or the $D_{1}$ are statistically significant at $p<0.0001$ (Table 3). (A) The LFD divides the complexity classes into the five subsets (clusters $1-5$ are marked by the numbers; compare Figure 4A). Those clusters contain carcinomas with different values of the LFD, and, therefore, with different intercellular connectivity. In particular, 10 carcinomas of the class $\mathrm{C} 1$ (black circles) and four carcinomas of the class C2 (red circles) compose a cluster two with the lowest values of both the $D_{0}$ and the LFD (Figure 4A), and the lowest aggressiveness. The values are close to those of benign prostatic hyperplasia. The cluster five represents high-grade carcinomas with the largest values of both the $D_{0}$ and the LFD, the largest complexity, and the largest tumor aggressiveness (red squares and blue diamonds). (B) The dimension $D_{1}$ is as strong classifier as the $D_{0}$, and divides the set of prostate carcinomas in the same classes of equivalence. Although the values of the Shannon entropy $H$ overlap between the classes, and, therefore, cannot play a role of the independent classifier, they characterize the spatial distribution of cancer cell nuclei in each class unequivocally if coupled with the $D_{0}$ or $D_{1^{-}}$ dimension. The coefficient of correlation for this relationship is 0.950. (C) The values of lacunarity $\lambda$ overlap between the classes of equivalence; however, a combination of this diversity measure with the $D_{0}$ or $D_{1}$-dimension allows the unequivocal classification of the underlying spatial distribution of cancer cell nuclei. The coefficient of correlation for this relationship is -0.902 . The statistical linearity of this relationship confirms in the independent way the existence of fractal structure in the spatial distribution of cancer cell cell nuclei. (D) Neither the Shannon entropy $H$ nor lacunarity $\lambda$ are strong classifiers. The values overlap between the classes of equivalence. A small subset of 
carcinomas of the class C7 overlaps with carcinomas of the class C5. This results from a lower number of cells in cellular infiltrates. However, this relationship is tri-linear. The coefficients of correlation for this relationship are $-0.911,-0.650$, and -0.922 for $H<0.7,0.7<H<0.9$, and $H>0.9$, respectively. The existence of tri-linearity suggests that some kind of qualitative transitions occur in natural history of prostate cancer between the class $\mathrm{C} 2$ and $\mathrm{C} 3$ as well as the class $\mathrm{C} 5$ and $\mathrm{C} 6$.

\section{A Relationship between the Global Capacity Dimension $D_{0}$ and Lacunarity $\lambda$ or the Global Information Dimension $D_{1}$ and Entropy $H$ or is Linear}

The relationship between the $D_{0}$ and $\lambda$ is given by linear equation (8):

$$
\lambda=2.0701-0.8145 D_{0}
$$

The coefficient of correlation $\mathrm{R}$ for that relationship was -0.902 . As it can be seen in Figure 3C, a given value of lacunarity $\lambda$ may be associated with different values of the $D_{0}$ dimension and different corresponding spatial distributions of cancer cell nuclei that belong to the neighbor classes of equivalence. Hence, the $\lambda$ value is not a good classifier. However, if this parameter is coupled with the $D_{0}$-value, it characterizes the spatial distribution of cancer cell nuclei unequivocally.

A similar capability possesses a pair $\left(D_{1}, H\right)$. As expected, there was a linear relationship between the global information fractal dimension $D_{1}$ and entropy $H$ given by the following statistical equation (9)

$$
H=-1.4280+1.3511 D_{1}
$$

Figure 3B demonstrates a distribution of carcinomas around the linear curve defined by Equation (9). The coefficient of correlation $R$ for that relationship was 0.950 . The linear relationship between the global information dimension as a complexity measure of cell nuclei clustering and the Shannon entropy as a diversity measure of information content validates in the independent manner the choice of the classes of 
equivalence. The $D_{1}$ is more accurate classifier than the Shannon entropy $H$ (Figure 3B). Some carcinomas of the class C3, C4, $\mathrm{C} 5$, C6, or $\mathrm{C} 7$ have entropy values that overlap with the values of the cases belonging to the adjacent classes. In particular, carcinomas of the class C7 are composed of cellular infiltrates only. Some of them have, however, a much lower number of infiltrating cells. Entropy of those carcinomas is lower, respectively (see Figure 3B, Class $\mathrm{C} 7$, light blue diamond). The values of lacunarity for those cases overlap with those for carcinomas of the class C5 (see Table 3 and Figure 3D).

A statistical relationship between lacunarity $\lambda$ and the Shannon entropy $H$ is more complex. This relationship is described by three linear equations that are defined for different intervalls of the $H$-values. The first one is given by equation (10)

$$
\lambda=1.0301-0.3057 \mathrm{H}
$$

and holds for $H=<0.7$. The coefficient of correlation $R=$ -0.911 .

The second one is given by Equation (11)

$$
\lambda=1.1625-0.5224 H
$$

and holds for $0.7<H<0.9$. The coefficient of correlation $R=$ -0.650 .

The third one is given by Equation (12)

$$
\lambda=1.7503-1.199 H
$$

and holds for $H>0.9$. The coefficient of correlation $R=-0.901$. Those relationships characterize well only the spatial distribution of cancer cell nuclei in prostate carcinomas with very low or very high diversity as measured by the Shannon entropy. Prostate carcinomas with intermediate diversity cannot be stratified with sufficient accuracy (compare Figures 3D,4B). The 
values of the pairs $(H, \lambda)$ overlap for many cases that belong to different classes of equivalence defined by the $D_{0}$ or the $D_{1}$ (Figure 3D). Figure 3A shows that the spatial distributions of cancer cell nuclei in many prostate carcinomas are unique as represented by different values of the pair $\left(D_{0}\right.$, LFD) (Figure $3 \mathrm{~A})$. Those values are not identical owing to multifractality. Simultaneously, many of those carcinomas have the same or almost identical values of the pair $\left(D_{1}, H\right)$ (Figure 3B) or $(H, \lambda)$ (Figure 3D). Two different fractal dimensions denote the existence of two different spatial distributions of cell nuclei. However, if the difference is not large, the Shannon entropy $H$ or lacunarity $\lambda$ may be identical owing to variability in cell numbers or their clustering.

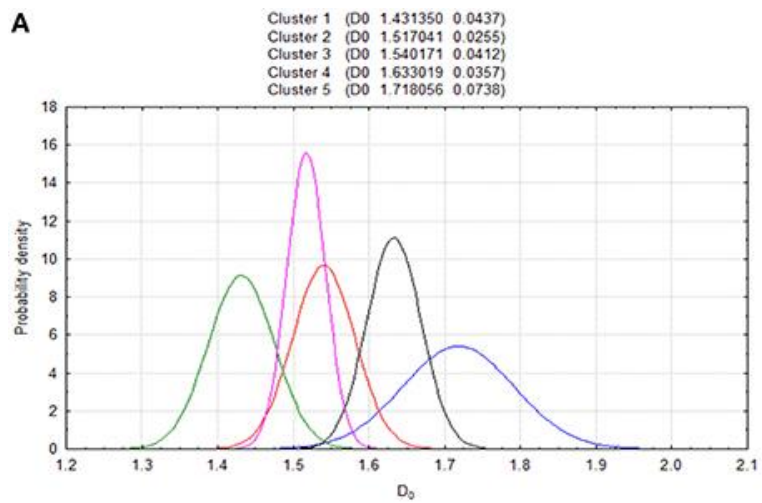

B

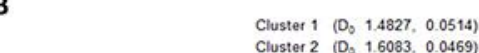

Cluster $2\left(\mathrm{D}_{0}, 1.6083,0.0469\right)$ Cluster $3(0,1.7166,00738)$

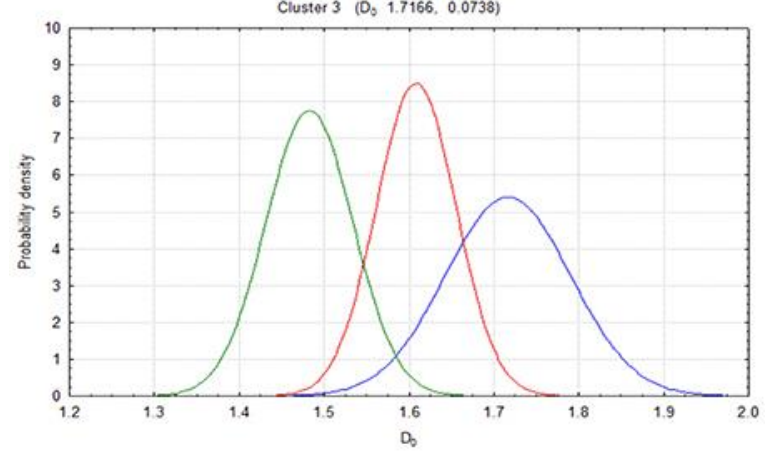

Figure 4: Results of cluster analysis for the test set of prostate carcinomas with the dimensions $D_{0}$ and LFD (A) (ANOVA $p<0.001, F_{D 0} 45799, \mathrm{~F}_{\mathrm{LFD}} 31935$ ) and the parameters $D_{0}$, LFD, LCFD, $H, \lambda$ (B) (ANOVA $p<0.001, F_{D 0} 75800$, 
$\left.F_{\mathrm{LFD}} 25289, F_{\mathrm{LCFD}} 13202, F_{H} 7925, F_{\lambda} 16102\right)$. The increment of the number of parameters from two to five reduces a number of clusters from seven to three. The objective analysis can identify in that way carcinomas with low-, intermediate-, and high complexity of the spatial distribution of cancer cell nuclei corresponding to low-, intermediate- or high tumor aggressiveness. The following conditions can be defined: for $D_{0}<1.5820$, LFD $<1.3$, LCFD $>1.5$, $H<0.7$ and $\lambda>0.8$, the class $\mathrm{C} 1$ or $\mathrm{C} 2$ contains low-complexity, low-grade carcinomas exclusively; for $D_{0}>1.6980$, LFD $>1.7644$, LCFD $>1.7051, H>$ 0.9 , and $\lambda<0.7$, the class $\mathrm{C} 6$ or $\mathrm{C} 7$ contains high-complexity, high-grade carcinomas only.

\section{The Co-Application of the Complexity and Diversity Measures in the Evaluation of the Spatial Distribution of Cancer Cell Nuclei in Prostate Carcinomas}

The cluster analysis by the $k$-means algorithm shows that the coapplication of both complexity and diversity measures, $D_{0}\left(D_{1}\right)$, LFD, LCFD, $H$, and $\lambda$ leads to a reduction of a number of clusters from seven to three (Figure 4B). Since low grade prostate carcinomas possess well-preserved glandular structure, the values of all the above measures but $\lambda$ are also low. The reduction of the number of clusters identifies some carcinomas of the complexity classes $\mathrm{C} 1$ and $\mathrm{C} 2$ as elements of the first cluster with the lowest values of those parameters, and, therefore, the lowest tumor aggressiveness. Similarly, one can define the quantitative criteria for carcinomas with the highest values of those parameters but $\lambda$, and the highest aggressiveness.

The mean LFD values do not increase along the complexity classes monotonically (see Figure 3A and Table 3). Rather, those values increase along each stratum, that is, a subset composed of the classes $\mathrm{C} 1$ and $\mathrm{C} 2$ or $\mathrm{C} 3$ and $\mathrm{C} 4$, or $\mathrm{C} 5$ and $\mathrm{C} 6$, or $\mathrm{C} 7$ (Table $3)$. The mean LFD values can be identical for carcinomas of different complexity classes.

The cluster analysis indicates five such subsets of prostate carcinomas characterized by the pair $\left(D_{0}, \mathrm{LFD}\right)$, in which the $D_{0}$ is a measure of the global spatial distribution of cancer cell nuclei and the local fractal dimension LFD is a measure of intercellular connectivity (see Figures 3A,4A). 
In the first subset, characterized by the parameters $D_{0}<1.5820$ and LFD $<1.35$, fourteen prostate carcinomas of the class $\mathrm{C} 1$ or C2 (low grade prostate carcinomas with mean PSA $<6.1 \mathrm{ng} / \mathrm{ml}$, standard deviation $1.2 \mathrm{ng} / \mathrm{ml}$, pT1b, pT1c, or pT2a, Gleason score 6, relatively homogeneous well-preserved glandular structure with glands distributed in tissue sparsely, without a clustering or crowding, none of those patients developed recurrence or metastases, or died owing to prostate cancer during the 5 years of follow-up) revealed very high intercellular connectivity. Those features are reflected by the value of the local connected fractal dimensions LCFD close to 1.5 (the scatter plot not shown), low values of entropy $H<0.7$ and high values of lacunarity $\lambda>0.8$ (compare Figures 3A-D). The low values of the fractal dimensions indicate that both global and local complexity in those carcinomas is low. In addition, the values of those fractal dimensions are quite close to the values obtained for benign prostatic hyperplasia (see Table 3). All those local and global features of the spatial distribution of cancer cell nuclei are associated with a low potential for growth and aggressiveness.

The second subset is composed of the low risk adenocarcinomas (mean PSA $8.3 \mathrm{ng} / \mathrm{ml}$, standard deviation $2.6 \mathrm{ng} / \mathrm{ml}$, Gleason score $6, \mathrm{pT} 1 \mathrm{~b}, \mathrm{pT} 1 \mathrm{c}$, or pT2a) stratified also to the classes $\mathrm{C} 1$ or C2. However, the second subset has the same values of the $D_{0}$, $D_{1}$, almost the same values of entropy $H<0.78$ as well as lacunarity $\lambda>0.78$, and $1.300<$ LFD $<1.750$. The values of the first four parameters denote that number of cells and their spatial configurations between those two sets are comparable. However, the values of the LFD indicate the existence of more advanced alterations in intercellular connectivity among adenocarcinomas of the second subset in spite of the preserved glandular structure, and, therefore, denote a higher risk of progression. Those LFD values denote very slow, yet active growth owing to the lower intercellular connectivity, and, therefore, increased probability of metastasizing in the course of time in comparison with the first subset. Those cancer patients belong to a "gray" zone, in which a follow-up without any additional therapy may work fine for some cases of the lower classes of complexity, such as $\mathrm{C} 1, \mathrm{C} 2$, or even for some intermediate-risk cases of the class $\mathrm{C} 3$ and $\mathrm{C} 4$ having the low values of the LFD. 
The third and the fourth subset comprise intermediate risk prostate cancers of the complexity class $\mathrm{C} 3, \mathrm{C} 4, \mathrm{C} 5$ (mean PSA $12.7 \mathrm{ng} / \mathrm{ml}$, standard deviation $2.2 \mathrm{ng} / \mathrm{ml}$, Gleason score 7 and 8 , pT2b-2c). In the third subset, there are carcinomas with $1.5820<$ $D_{0}<1.6980$ and the LFD $<1.8$. Prostate carcinomas of the fourth subset have the same values of the $D_{0}$ as in the third set, but the LFD $>1.8$ (see Figure 3A) at $0.65<H<0.85$, and $0.7<$ $\lambda<0.82$ (Figures 3B,C). Surprisingly, there are two adenocarcinomas of the complexity class $\mathrm{C} 2$ in that subset. Their mean LFD value approximate the value of 1.9, that is typical of prostate carcinomas without glandular structure and with the increased potential to metastasize. In spite of the stratification to the low class of complexity owing to the $D_{0}$-value, such carcinomas with very low intercellular connectivity may have a high metastatic potential.

The fifth subset comprises high risk prostate cancers (mean PSA $24.3 \mathrm{ng} / \mathrm{ml}$, standard deviation $3.1 \mathrm{ng} / \mathrm{ml}$, Gleason score 9-10, stage T3a, the largest ratio of prostate carcinoma related death or metastasis formation within 5 years from the surgical treatment 0.442) of the complexity class C6 or C7 with $D_{0}>1.6980$ and the LFD $>1.8, H>0.9$, and $\lambda<0.7$. The large values of the $D_{0}$ and $H$ indicate a significantly increased cell number owing to the intense cell proliferation and rapid growth. Since images are covered almost completely by cancer cells, and become almost symmetrical, the $\lambda$-values are low. The high values of the LFD indicate the existence of a very low intercellular connectivity. Cells fill up the entire available tissue space without any clustering, and, therefore, have the largest potential for metastasis formation.

\section{Discussion}

The classes of equivalence defined by the cut-off values of the global capacity dimension $D_{0}$ reported previously [66] have been validated. The other measures but the $D_{1}$ failed to define the same classes of equivalence. However, the co-application of all the measures allowed the formulation of two quantitative criteria identifying low or high aggressive prostate carcinomas. 
A novel, quantitative strategy for the evaluation of tumor aggressiveness excludes the automated diagnosis of prostate carcinoma. The complexity or diversity measures were not able to discriminate between the spatial distribution of cell nuclei in benign prostatic hyperplasia, a common lesion in human prostate, and carcinomas with a well-preserved glandular structure (see Table 3). This approach implies a necessity to examine the entire prostate carefully, what does not seem to be the case in the routine practice [84]. Indeed, the largest value of the fractal dimension $D_{0}$ or $D_{1}$ determines the stratification of carcinomas into a given class of equivalence.

Results of this study suggest that the number of the subjective Gleason grades of tumor aggressiveness could be decreased from seven to three without a loss of clinically important information, that is, to the low, intermediate, or high grade. The identical reduction could be done for a number of the objective classes of equivalence. Table 1 indicates that pathologists can distinguish quite well between the relatively homogeneous patterns with the Gleason score $3+3,4+4$, or $5+5$ (see the reference set). However, heterogeneous patterns defined as the Gleason score 3+4, 4+3, $4+5$, or $5+4$ were classified with much greater inaccuracy (see the test set). The classes of equivalence defined by the cut-off $D_{0}$-values enable the quantitative evaluation of tumor aggressiveness of borderline cases with mixed tissue architecture; a frequent problem in pathology of prostate carcinoma [85]. It should be noticed that the discrepancy between the subjective Gleason classification and the classification according to complexity of the spatial distribution of cancer cell nuclei was on average about 53\% in this study; a value that is in the range of the intra- and interobserver variability $([12-20,85]$.

The classes of equivalence, i.e., the complexity classes defined by the cut-off values of the $D_{0}$ dimension (or the $D_{1}$ dimension) were validated by analyzing the test set of carcinomas with sensitivity, specificity, and AUC equal 1.0 [66]. This set was larger and independent from the set analyzed in the previously published study [66]. The values of the global capacity fractal dimension $D_{0}$ are identical with those previously reported [66] 
(see Tables 2, 3, Figure 3). Those two dimensions appear to be the best classifiers of prostate carcinomas according to the spatial distribution of cancer cell nuclei. There is a very good correlation between the values of the $D_{0}$ and the $D_{1}$ (correlation coefficient $R=0.962$ ). Those results were to be expected since both sets comprised different numbers of carcinomas with the similar patterns of tumor growth. As expected, the $D_{1}$-values correlate very well with the values of the Shannon entropy $H$; a diversity measure of both randomness in the spatial distribution of cell nuclei and information content in images of a given class $(R=0.950$; see Figure 3B). There also is some correlation between the values $H$ and $\lambda$ that changes depending on the $H$ intervals (compare Equations 10-12). The $\lambda$ values overlap between classes (see Figure 3D), and, therefore, cannot be applied for stratification as the independent classifier. The overlapping results from the non-bijective relationship between some configurations of cell nuclei and the measures applied [66]. In particular, two borderline spatial distributions of cancer cell nuclei may have identical values of the complexity measures, but different values of the diversity measures. In spite of that weakness, the diversity measures help to identify carcinomas with low or high diversity corresponding to carcinomas with the low or high grade (see Figures 3B,C). Each of those measures characterizes quantitatively different aspects of the spatial distribution of cancer cell nuclei in images of prostate carcinomas. While the $D_{0}$ or the $D_{1}$ enables the stratification of carcinomas into the seven classes of equivalence, that remain unchanged if the LFD, the LCFD, the Shannon entropy $H$ or $\lambda$ are added to the class characteristics (see Figure 3), the coapplication of all the above-studied measures allows the stratification of the same carcinomas into just three distinct clusters with low tumor aggressiveness (class $\mathrm{C} 1, \mathrm{C} 2$, and some carcinomas of the class $\mathrm{C} 3$ ), intermediate one (some carcinomas of the class C3, C4, and C5) or high one (class C6 and C7) (compare Figure 4). The appropriate quantitative criteria are defined in the Sections Results: A Relationship between the Global Capacity Fractal Dimension $D_{0}$ and Lacunarity $\lambda$ or the Global Information Fractal Dimension $D_{1}$ and Entropy $H$ or is Linear; The Co-Application of the Complexity and Diversity 
Measures in the Evaluation of the Spatial Distribution of Cancer Cell Nuclei in Prostate Carcinomas.

The presented data indicate some change in intercellular connectivity at the interface between classes $\mathrm{C} 2$ and $\mathrm{C} 3$ as well as C5 and C6 as measured by the LFD; a complexity measure of cellular connectivity, that is, a measure of strength of intercellular interactions (Table 3, Figure 3A). This change can also be seen in the scatter plot $(H, \lambda)$ (Figure 3D). The relationship between the Shannon entropy $\mathrm{H}$ and lacunarity $\lambda$; a diversity measure of cell clustering also changes at the interface between the above-mentioned classes and depends on the $\mathrm{H}$ values $(R=-0.911$ for $H<0.7, R=-0.650$ for $0.7<H<0.9$, and -0.901 for $H>0.9$ ). Those changes correspond with the enhanced dynamics of tumor growth at the interface between low and intermediate risk cancers as well as intermediate and high risk cancers. It is possible that all those changes result from some genetic instability that gains influence on growth dynamics at some specific check-points in natural history of prostate cancer. Second, results of the analysis point out that prostate carcinomas with the spatial distributions of cancer cell nuclei holding the conditions $D_{0}<1.5820$, LFD $<1.3$, and LCFD $>1.5$ are true low grade carcinomas. Those carcinomas possess a wellpreserved, relatively homogeneous glandular structure that resembles the structure of the normal prostatic glands. There is some intermediate cell clustering at the low values of the LFD and, therefore, high intercellular connectivity in cell populations of those adenocarcinomas (Figure 3A). Hence, there is no high growth potential in those cellular populations. If $D_{0}>1.6980$, LFD > 1.7644, and LCFD > 1.7051, then tumor aggressiveness is large. Cancer cells infiltrate the entire available tissue space, have low intercellular connectivity, do not interact each other, and compose passively multiple cell clusters; the overcrowding effect. This constellation of dimensions indicates the existence of a large growth potential in a cellular population. The cases with intermediate conditions compose a gray zone. Some of those carcinomas may do well under follow-up, especially those with the lower values of the LFD and LCFD. The other cases will certainly require some kind of therapy in the course of time. It is interesting, however, that there is no carcinoma with the low 
values of both local fractal dimensions. If the LFD increases, then intercellular connectivity decreases, and cells lose their capability to interact with each other. Therefore, cells in some carcinomas including those of the class $\mathrm{C} 1, \mathrm{C} 2, \mathrm{C} 3$, and $\mathrm{C} 4$ do not create clusters (the lower values of the LCFD), while many others still do (the larger values of the LCFD). The loss of capability for cell clustering at low values of the LCFD may also denote both the increased growth potential and risk of metastasis formation.

The increasing values of the $D_{0}$ dimension denote that cellular proliferation in a given carcinoma is very extensive, and more and more cancer cells fill up the available tissue space. If cancer cell gather in some kind of glands or gland-like objects, the $D_{0^{-}}$ values are lower than if there dominate cellular infiltrates in the image (compare the values of the $D_{0}$ for the classes $\mathrm{C} 1, \mathrm{C} 2$ or C3 with those for the classes $\mathrm{C} 6$ or $\mathrm{C} 7$ in Table 3). The larger is the value of the $D_{0}$ the higher is the complexity class and the corresponding subjective grade. The higher is the complexity class the higher is a number of cancer cells present in the image of prostate carcinoma. Cancer cells lose in the course of natural tumor evolution their cluster organization in a form of glands (high values of the $D_{1}$, the LFD, and the LCFD and low values of $\lambda$ ), become more randomly distributed (high values of $H$ ), and reveal lower connectivity (high values of the LFD and the LFCD) (compare Figures 3A-D). The risk of local progression or metastasis formation is increased in those spatial distributions of cancer cell nuclei.

The existence of both the global and local fractal dimensions for a given spatial distribution of cancer cell nuclei implies that that spatial distribution underlies a scale-dependent power law. The existence of the power law means that the spatial distribution of cancer cell nuclei undergoes somehow ordered rather than utterly random changes in the course of natural history of the prostatic tumorigenesis. That order of events appears to comprise the gradual loss of intercellular connectivity in carcinomas of the higher complexity classes (see Table 3 and Figure 3). This may occur owing to the loss of expression of adhesion molecules or some other molecules with biologically important functions 
leading to alterations of intercellular interactions ([86-89]. For the majority of carcinomas, the values of both the global and local fractal dimension are not identical (see Table 3 and Figure 3 ). That difference between the values of the global and local fractal dimensions indicates the existence of multifractals in the spatial distributions of cancer cell nuclei, that is, fractals that scale with multiple scaling rules and hold the condition $D_{0}>D_{1}$ $>D_{2}$ (reviewed in Lopes and Betrouni, [90]). Multifractality suggests that the final spatial distribution of cancer cells depends on both a variety of molecular events and long-range phenomena such as diffusion. The interactions may occur at different levels of the hierarchical tissue dynamic system [73,76,77]. Those complex phenomena compose a new world to be explored.

Two diversity measures CV-LFD and CV-LCDF do not play a role in the stratification process (see Table 3 ). Those diversity measures apply to differences in measurements of the local fractal dimensions within each complexity class [91]. There is no monotonic increment of the values of those parameters that might enable the stratification process. However, entropy, the other diversity measure, appears to be useful in the additional characterization of the spatial distribution of cell nuclei (Equations 2, 4, 5 and Figure 3B). Even though the local fractal dimensions in each complexity class that relate to single cancer cell nuclei are not diverse, cancer cells as a population form diverse dynamic spatial structures of the higher order, such as cell clusters (see Figure 3A). Those cell clusters can be characterized locally by the values of the local connected fractal dimension, entropy, and lacunarity. In addition, the LFD identifies cells with the lowest intercellular connectivity, and, therefore, having the maximal metastatic potential (see Figure 3A; [73-78]). Computer simulations of a model considering the evolution of cooperation in a spatial setting obtained by Nowak and May show clearly the existence of a relationship between emergent diversity and complexity $[92,93]$. The existence of that relationship in prostate carcinomas can be seen in equation 5 and Figure 3B.

To summarize, the following findings must be reiterated. First, the classes of equivalence for prostate carcinomas by the cut-off 
$D_{0}$-values were validated. Second, no other measure studied but the $D_{1}$ can define the same classes of equivalence. Third, carcinomas with low complexity of the spatial distribution of cancer cell nuclei reveal low biological aggressiveness, and vice versa. Some pairs of the parameters $\left(D_{0}, \mathrm{LFD}\right),\left(D_{0}, H\right)$ or $\left(D_{0}, \lambda\right)$ or $\left(D_{1}, \mathrm{LFD}\right),\left(D_{1}, H\right)$ or $\left(D_{1}, \lambda\right)$ characterize well complexity of the spatial distribution of cancer cell nuclei in each class of equivalence. The tri-linear course of the relationship $(H, \lambda)$ suggests that among carcinomas of the classes $\mathrm{C} 2$ and $\mathrm{C} 3$ as well as $\mathrm{C} 5$ and $\mathrm{C} 6$ occurs some kind of a qualitative transition that implicates changes in tumor aggressiveness. Fourth, the coapplication of all complexity and diversity measures reduces a number of clusters, but still enables the identification of carcinomas with low or high complexity of the spatial distribution of cancer cell nuclei. Those findings suggest the number of both the subjective Gleason grades and the objective classes of equivalence could be decreased from seven to three.

\section{References}

1. Humphrey PA. Grading of prostatic carcinoma. In Prostate Pathology. Chicago: ASCP Press. 2003; 338-374.

2. Gleason DF. Histologic gradisng and clinical staging of prostatic carcinoma, in Urologic Pathology. M Tannenbaum, editor. Philadelphia: Lea and Febiger. 1997; 171-198.

3. Humphrey PA. Gleason grading and prognostic factors in carcinoma of the prostate. Mod. Pathol. 2004; 17: 292-306.

4. Epstein JI, Allsbrook WC, Amin MB, Egevad LL, the ISUP Grading Committee. The 2005 International Society of Urological Pathology (ISUP) consensus conference on Gleason grading of prostatic carcinoma. Am. J. Surg. Pathol. 2005; 29: 1228-1242.

5. Epstein JI. An update of the Gleason Grading System. J. Urol. 2010; 183: 433-440.

6. Lotan TL, Epstein JI. Clinical implications of changing definitions within the Gleason grading system. Nat. Rev. 2010; 7: 136-142.

7. Fine SW, Amin MB, Berney DM, Bjartell A, Egevad L, et al. A contemporary update on pathology reporting for 
prostate cancer: biopsy and radical prostatectomy specimens. Eur. Urol. 2012; 62: 20-39.

8. Epstein JI, Walsh PC, Carmichael M, Brendler CB. Pathologic and clinical findings to predict tumor extent of nonpalpable (stage T1c) prostate cancer. JAMA. 1994; 271: 368-374.

9. D'Amico AV, Wu Y, Chen MH, Nash M, Renshaw AA, et al. Pathologic findings and prostate specific antigen outcome after radical prostatectomy for patients diagnosed on the basis of a single microscopic focus of prostate carcinoma with a Gleason score </=7. Cancer. 2010; 89: 1810-1817.

10. Boccon-Gibod LM, Dumonceau O, Toublanc M, Ravery V, Boccon-Gibod LA. Micro-focal prostate cancer: a comparison of biopsy and radical prostatectomy specimen features. Eur. Urol. 2005; 48: 895-899.

11. Montironi R, Lopez-Bertran A, Cheng L, Montorsi F, Scarpelli M. Central prostate pathology review: should it be mandatory? Eur. Urol. 2013; 64: 99-203.

12. Nguyen PL, Schultz D, Renshaw AA, Volmer RT, Welch $\mathrm{WR}$, et al. The impact of pathology review on treatment recommendations for patients with adenocarcinoma of the prostate. Urol. Oncol. Semin. Orginal Invest. 2004; 22: 295299.

13. van der Kwast TH, Evans A, Lockwood G, Tkachuk D, Bostwick DG, et al. Variability in diagnostic opinion among pathologists for single small atypical foci in prostate biopsies. Am. J. Surg. Pathol. 2010; 34: 169-177.

14. McKenney JK, Simko J, Bonham M, True LD, Troyer D, et al. Canary/early detection research network prostate active surveillance study investigators. the potential impact of reproducibility of gleason grading in men with early stage prostate cancer managed by active surveillance; a multiinstitutional study. J. Urol. 2011; 186: 465-469.

15. Netto GJ, Eisenberger M, Epstein JI. Interobserver variability in histologic evaluation of radical prostatectomy between central and local pathologists: findings of TAX 3501 multinational clinical trial. Urology. 2011; 77: 11551160.

16. Egevad L, Ahmad AS, Algaba F, Berney DM, BocconGibod L, et al. Standardization of Gleason grading among 
337 European pathologists. Histopathology. 2013; 62: 247256.

17. Berney DM, Algaba F, Camparo P, Compérat E, Griffiths D, et al. The reasons behind variation in Gleason grading of prostatic biopsies: areas of agreement and misconception among 266 European pathologists. Histopathology. 2011; 64: 405-411.

18. McLean M, Srigley J, Banerjee D, Warde P, Hao Y. Interobserver variation in prostate cancer Gleason scoring: are there implications for the design of clinical trials and treatment strategies? Clin. Oncol. 1997; 9: 222-225.

19. Allsbrook WC, Mangold KA, Johnson MH, Lane RB, Lane $\mathrm{CG}$, et al. Interobserver reproducibility of Gleason grading of prostatic carcinoma: general pathologist. Hum. Pathol. 2001; 32: 81-88.

20. Scott Lucia M, Bostwick DG, Somerville MC, Fowler IL, Rittmaster RS. Comparison of classic and international society of urological pathology 2005 modified gleason grading using needle biopsies from the reduction by dutasteride of prostate cancer events (REDUCE) Trial. Archiv. Pathol. Lab. Med. 2013; 137: 1740-1746.

21. McDunn JE, Li Z, Adam KP, Neri BP, Wolfert RL, et al. Metabolomic signatures of aggressive prostate cancer. Prostate. 2013; 73: 1547-1560.

22. Pin E, Fredolini C, Petricoin EF. The role of proteomics in prostate cancer research: biomarker discovery and validation. Clin. Biochem. 2013; 46: 524-538.

23. Turkbey B, Choyke PL. Multiparametric MRI and prostate cancer diagnosis and risk stratification. Curr. Opin. Urol. 2012; 22: 310-315.

24. Klein E. Prostate cancer. In: ME Ross, editor. Risk stratification and choice of initial treatment. 2014. Available online at: http://www.uptodate.com/contents/prostatecancer-risk-stratification-and-choice-of-initial-treatment

25. Mottet N, Bellmunt J, Briers E, van den Bergh RCN, Bolla $\mathrm{M}$, et al. Guidelines on Prostate Cancer. European Association for Urology. 2015; 30-97. Available online at: http://uroweb.org/wp-content/uploads/09-ProstateCancer_LR.pdf 
26. National Comprehensive Cancer Network (NCCN). NCCN Clinical Practice Guidelines in Oncology. 2015. Available online

at: http://www.nccn.org/professionals/physician_gls/f_guideline s.asp

27. Partin AW, Kattan MW, Subong ENP, Walsh PC, Wojno $\mathrm{KJ}$, et al. A combination of prostate-specific antigen, clinical stage, and Gleason score to predict pathological stage of localized prostate cancer. A multi-institutional Update. JAMA. 1997; 277: 1445-1451.

28. Stamey TA, Kabalin JN, McNeal JE, Johnstone IM, Freiha $\mathrm{F}$, et al. Prostate specific antigen in the diagnosis and treatment of adenocarcinoma of the prostate: II. Radical prostatectomy treated patients. J. Urol. 1989; 141: 10761083.

29. D'Amico AV, Chen MH, Roehl KA, Catalona WJ. Preoperative PSA velocity and the risk of death from prostate cancer after radical prostatectomy. N. Engl. J. Med. 2004; 351 125-135.

30. D'Amico AV, Chen MH, Roehl KA, Catalona WJ. Identifying patients at risk for significant versus clinically insignificant postoperative prostate-specific antigen failure. J. Clin. Oncol. 2005; 23: 4975-4979.

31. Klotz L, Zhang L, Lam A, Nam R, Mamedov A, et al. Clinical results of long-term follow-up of a large, active surveillance cohort with localized prostate cancer. J. Clin. Oncol. 2010; 28: 126-131.

32. Thompson IM, Ankerst DP, Chi C, Goodman PJ, Tangen $\mathrm{CM}$, et al. Assessing prostate cancer risk: Results from the prostate cancer prevention trial. J. Natl. Cancer Inst. 2006; 98: 529-534.

33. ElShafei A, Li YH, Hatem A, Moussa AS, Ethan V, et al. The utility of PSA velocity in prediction of prostate cancer and high grade cancer after an initially negative prostate biopsy. Prostate. 2013; 73: 1796-1802.

34. Wu JN, Fish KM, Evans CP, Devere White RW, Dall'Era MA. No improvement noted in overall or cause-specific survival for men presenting with metastatic prostate cancer over a 20-year period. Cancer. 2014; 120: 818-823. 
35. Prout GR, Heaney JA, Griffin PP, Daly JJ, Shipley WU. Nodal involvement as a prognostic indicator in patients with prostatic carcinoma. J. Urol. 1980; 124: 226-231.

36. Petros JA, Catalona WJ. Lower incidence of unsuspected lymph node metastases in 521 consecutive patients with clinically localized prostate cancer. J. Urol. 1992; 147: 1574-1575.

37. Miller DC, Gruber SB, Hollenbeck BK, Montie JE, Wei JT. Incidence of initial local therapy among men with lower-risk prostate cancer in the United States. J. Natl. Cancer Inst. 2006; 98: 1134-1141.

38. Danzig MR, McKiernan JM. Overtreatment of men with early-stage prostate cancer and limited life expectancy. Cancer. 2014; 120: 3592-3594.

39. Daskivich TJ, Lai J, Dick AW, Setodji CM, Hanley JM, et al. Variation in treatment associated with life expectancy in a population-based cohort of men with early-stage prostate cancer. Cancer. 2014; 120: 3642-3650.

40. Dall'Era MA, Konety BR, Cowan JE, Shinohara K, Stauf F, et al. Active surveillance for the management of prostate cancer in a contemporary cohort. Cancer. 2008; 112: 26642670.

41. Albertsen PC. Treatment of localized prostate cancer: when is active surveillance appropriate? Nat. Rev. Clin. Oncol. 2010; 7: 394-400.

42. Klotz L. Active surveillance versus radical treatment for favorable-risk localized prostate cancer. Curr. Treat. Options Oncol. 2006; 7: 355-362.

43. Denis L. Active Surveillance in prostate cancer. Belg. J. Med. Oncol. 2007; 1: 3-7.

44. Albertsen PC, Hanley JA, Finer J. 20-year outcomes following conservative management of clinically localized prostate cancer. JAMA. 2005; 293: 2095-2101.

45. Cooperberg MR, Cowan JE, Hilton JF, Reese AC, Zaid HB, et al. Outcomes of active surveillance for men with intermediate-risk prostate cancer. J. Clin. Oncol. 2011; 29: 228-234.

46. Cooperberg MR. Long-term active surveillance for prostate cancer: answers and questions. J. Clin. Oncol. 2015; 33: 238-240. 
47. Klotz L, Vesprini D, Sethukavalan P, Jethava V, Zhang L, et al. Long-term follow-up of a large active surveillance cohort of patients with prostate cancer. J. Clin. Oncol. 2014; 33: 272-277.

48. Womble PR, Montie JE, Ye Z, Linsell SM, Lane BR, et al. A contemporary use of initial active surveillance among men in Michigan with low-risk prostate cancer. Eur. Urol. 2015; 67: 44-50.

49. Heidenreich A, Bastian PJ, Bellmunt J, Bolla M, Joniau S, et al. European association of urology. EAU guidelines on prostate cancer. Part II: Treatment of advanced, relapsing, and castration-resistant prostate cancer. Eur. Urol. 2014; 65: 467-479.

50. Lu-Yao GL, Albertsen PC, Moore DF, Shih W, Lin Y, et al. Fifteen-year survival outcomes following primary androgendeprivation therapy for localized prostate cancer. JAMA Int. Med. 2014; 174: 1460-1467.

51. Altunbay D, Cigir C, Sokmensuer C, Gunduz-Demir C. Color graphs for automated cancer diagnosis and grading. IEEE Trans. Biomed. Eng. 2010; 57: 665-674.

52. Naik S, Doyle S, Agner S, Madabhushi A, Feldman M, Tomaszeweski J. Automated gland and nuclei segmentation for grading of prostate and breast cancer histopathology. In Proceedings of IEEE ISBI. Paris: IEEE. 2008; 284-287.

53. Loeffler M, Greulich L, Scheibe P, Kahl P, Shaikhibrahim Z, et al. Classifying prostate cancer malignancy by quantitative histomorphometry. J. Urol. 2012; 187: 1867-1875.

54. Nguyen K, Sabata B, Jain AK. Prostate Cancer grading: gland segmentation and structural features. Pattern Recognit. Lett. 2012; 33: 951-961.

55. Doyle S, Hwang M, Shah K, Madabhushi A, Feldman M, Tomaszeweski J. Automated grading of prostate cancer using architectural and textural image features. In Proceedings of IEEE ISBI. Washington, DC: IEEE. 2007; 1284-1287.

56. Jafari-Khouzani K, Soltanian-Zadeh H. Multiwavelet grading of pathological images of prostate. IEEE Trans. Biol. Eng. 2003; 50: 697-704. 
57. Candes EJ, Demanet L, Donoho DL, Ying L. Fast discrete curvelet transform. Multiscale Model. Simulations. 2006; 5: 861-899.

58. Huang PW, Lee $\mathrm{CH}$. Automatic classification for pathological prostate images based on fractal analysis. IEEE Trans. Med. Imaging. 2009; 28: 1037-1050.

59. Hong-Jun Y, Ching-Chung L, Christudass C, Veltri R, Epstein JI, et al. Cardinal multiridgelet-based prostate cancer histological image classification for Gleason grading, in Proceedings IEEE BIBM. Atlanta, GA: IEEE. 2012; 315320.

60. Veltri R, Christhunesa W, Christudass S, Isharwal S. Nuclear morphometry, nucleomics and prostate cancer progression. Asian J. Androl. 2012; 14: 375-384.

61. Lopez M, Again S. A new set of wavelet- and fractals-based features for Gleason grading of prostate cancer histopathology images. In Proceedings SPIE-IS\&T Electronic Imaging. Burlingame. 2013; 8655: 865516.

62. Tabesh A, Kumar VP, Pang HY, Verbel D, Kotsianti A, et al. Automated prostate cancer diagnosis and Gleason grading of tissue microarrays, in Proceedings SPIE, Vol. 5747. San Diego, CA. 2005; 58-70.

63. Tabesh A, Teverovskiy M, Pang HY, Kumar VP, Verbel D, et al. Multifeature Prostate cancer diagnosis and gleason grading of histological images. IEEE Trans. Med. Imaging. 2007; 26:1366-1378.

64. Waliszewski P. Fractals and Complexity. Wrocław: Format. 2013.

65. Waliszewski P, Wagenlehner F, Kribus S, Schafhauser W, Weidner W, et al. Objektivierung des tumorgradings bei prostatakarzinomen anhand der globalen und lokalen fraktalen dimensionen. Urol. A. 2014; 53: 1504-1511.

66. Waliszewski P, Wagenlehner F, Gattenloehner S, Weidner W. On the Relationship between tumor structure and complexity of the spatial distribution of cancer cell nuclei; a fractal geometrical model of prostate carcinoma. Prostate. 2015; 75: 399-414.

67. Engelking R. Dimension Theory. Amsterdam: North Holland Publishing Company and Warsaw; PWN. 1978. 
68. Czyz J. Paradoxes of Measures and Dimensions Originating in Felix Hausdorff's Ideas. Singapore: World Scientific Pub Co Inc. 1994.

69. Vicsek T. Fractal Growth Phenomena. 2nd edn. Singapore: World Scientific. 1992.

70. Kinsner W. A unified approach to fractal dimensions. Int. J. Cogn. Inform. Nat. Intell. 2007; 1: 26-45.

71. Baker GL, Gollub JP. Chaotic Dynamics: an Introduction. Cambridge: Cambridge University Press. 1996.

72. Grassberger P, Proccacia I. Measuring the strangeness of strange attractors. Phys. D. 1983; 9: 189-208.

73. Waliszewski P. Complexity dynamic cellular network, and tumorigenesis. Pol. J. Pathol. 1993; 48: 235-241.

74. Waliszewski P. A principle of fractal-stochastic dualism and Gompertzian dynamics of growth and self-organization. Biosystems. 2005; 82: 61-73.

75. Waliszewski P. A principle of fractal-stochastic dualism, couplings, complementarity and growth. CEAI. 2009; 11: 45-52.

76. Waliszewski P, Molski M, Konarski J. On the holistic approach in cellular and cancer biology: nonlinearity, complexity, and quasi-determinism of the dynamic cellular network. J. Surg. Oncol. 1998; 68: 70-78.

77. Waliszewski P, Molski M, Konarski J. On the relationship between fractal geometry of space and time in which a system of interacting cells exists and dynamics of gene expression. Acta Biochim. Pol. 2001; 48: 209-220.

78. Waliszewski $\mathrm{P}$, Konarski J. Tissue as a self-organizing system with fractal dynamics. Adv. Space Res. 2001; 28: $545-548$.

79. Landini G, Murray I, Misson GP. Local connected fractal dimensions and lacunarity analyses of $60^{\circ}$ Fluorescein angiograms. Invest. Ophthalmol. Vis. Sci. 1995; 36: 2749 2755.

80. Sethna JP. Satistical Mechanics. Entropy, Order Parameters, and Complexity. Oxford: Clarendon Press. 2011.

81. Plotnick R, Gardner R, Hargrove W, Perlmutter M, Prestegaard K. Lacunarity analysis: a general technique for the analysis of spatial patterns. Phys. Rev. E. 1996; 53: 5461-5468. 
82. Kristiansen G, Epstein JI. Immunohistochemistry in Prostate Pathology. DAKO. 2014. Available online at http://www.dako.com/de/ihc-prostate-pathology.pdf

83. Hill T, Lewicki P. Statistics: Methods and Applications. A Comprehensive Reference for Science, Industry and Data Mining. Tulsa: Statsoft Inc. 2006.

84. True LD. Surgical pathology examination of the prostate gland. practice survey by american society of clinical pathologists. Am. J. Clin. Pathol. 1994; 102: 572-579.

85. Dong F, Wang C, Farris AB, Wu S, Lee H, et al. Impact on the clinical outcome of prostate cancer by the 2005 international society of urological pathology modified Gleason grading system. Am. J. Surg. Pathol. 2012; 36: 838843.

86. Busch TA, Hanssen TA, Wagener C, Oebrink B. Downregulation of CEACAM1 in human prostate cancer: correlation with loss of cell polarity, increased proliferation rate, and Gleason grade 3 to 4 transition. Hum. Pathol. 2002; 33: 290-298.

87. Huang L, Pu Y, Yang Hu W, Birch L, Luccio-Camelo D, et al. The role of Wnt5a in prostate gland development. Dev. Biol. 2009; 328: 188-199.

88. Mathieu R, Evrard B, Fromont G, Rioux-Leclercq N, Godet $\mathrm{J}$, et al. Expression screening of cancer/testis genes in prostate cancer identifies nr6al as a novel marker of disease progression and aggressiveness. Prostate. 2014; 73: 11031114.

89. Schrecengost R, Knudsen KE. Molecular pathogenesis and progression of prostate cancer. Semin. Oncol. 2013; 40: 244-258.

90. Lopes R, Betrouni N. Fractals and multifractal: a review. Med. Image Analysis. 2009; 13: 634-649.

91. Page SE. Diversity and Complexity. Princeton: Princeton University Press. 2011.

92. Nowak MA, May RM. Evolutionary games and spatial chaos. Nature. 1992; 359: 826-829.

93. Nowak MA, May RM. The spatial dilemmas of evolution. Int. J. Bifurc. Chaos. 1993; 3: 35-78. 